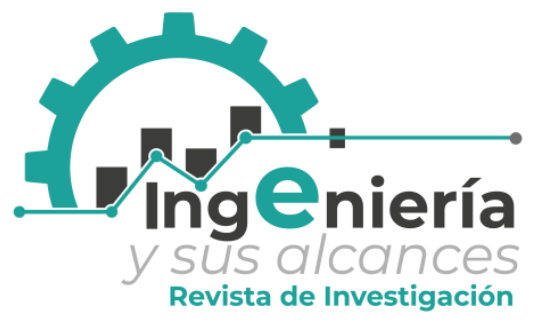

Ingeniería y sus alcances, Revista de Investigación Https://doi.org/10.33996/revistaingenieria.v4i10.67 Septiembre - diciembre 2020 Volumen 4 / No. 10 ISSN: $2664-8245$ ISSN-L: $2664-8245$ pp. $208-232$

\title{
Deflexión en arcos usando métodos energéticos
}

\author{
Deflection in arcs using energetic methods
}

Deflexão em arcos usando métodos energéticos

\author{
Miranda Casazola Dayan Esmirna \\ dayanmca8@gmail.com \\ ORCID 0000-0003-0802-3402 \\ Universidad Autónoma Tomas Frías, Bolivia
}

Artículo recibido mayo 2020 | Arbitrado en julio 2020 | Publicado en septiembre 2020

RESUMEN

Los avances en la construcción de puentes consideran técnicas de diseño de arcos gracias a sus ventajas técnicas. Esta investigación se propuso determinar la mejor opción para abordar el problema del cálculo de la deflexión en arcos circulares de hormigón para un puente; mediante un enfoque cuantitativo de corte transversal con alcances descriptivo y aplicado. Desde una perspectiva teórica se aplicó el método bibliográfico y para el cálculo matemático se aplicaron los métodos energéticos $y$, para comparar los hallazgos, el método de elementos finitos mediante la herramienta software SAP 2000. Como resultado de la aplicación de los métodos energéticos se obtuvo 10,21mm de deflexión total; asimismo, aplicando el programa Sap2000 se obtuvo $12,48 \mathrm{~mm}$ de deflexión total. Se concluyó que los métodos energéticos son más eficientes en el cálculo de deflexiones en estructuras curvas, particularmente, arcos circulares.

Palabras clave: Métodos energéticos; arcos; puentes; diseño estructural; elementos finitos

ABSTRACT

RESUMO

Advances in bridge construction consider arch design techniques due to their technical advantages. This research set out to determine the best option to address the problem of calculating deflection in concrete circular arches for a bridge, using a cross-sectional quantitative approach with descriptive and applied scopes. From a theoretical perspective, the bibliographic method was applied. For the mathematical calculation, the energetic methods were applied and, to compare the findings, the finite element method using the SAP 2000 software tool. As a result of the application of the energetic methods $10.21 \mathrm{~mm}$ of total deflection was obtained; Likewise, applying the Sap2000 program, $12.48 \mathrm{~mm}$ of total deflection was obtained. It was concluded that energetic methods are more efficient in calculating deflections in curved structures, particularly circular arches.

Key words: Energy methods; bows; bridges; structural design; finite elements
Os avanços na construção de pontes consideram as técnicas de projeto de arco devido às suas vantagens técnicas. Esta pesquisa teve como objetivo determinar a melhor opção para resolver o problema do cálculo da deflexão em arcos circulares de concreto para uma ponte; por meio de uma abordagem quantitativa transversal com escopos descritivo e aplicado. Do ponto de vista teórico, foi aplicado o método bibliográfico e para o cálculo matemático foram aplicados os métodos energéticos e, para comparação dos achados, o método dos elementos finitos utilizando a ferramenta do software SAP 2000. Como resultado da aplicação dos métodos energéticos, obteve-se 10,21mm de deflexão total; Da mesma forma, aplicando o programa Sap2000, obteve-se $12,48 \mathrm{~mm}$ de deflexão total. Concluiu-se que os métodos energéticos são mais eficientes no cálculo de deflexões em estruturas curvas, principalmente em arcos circulares.

Palavras-chave: Métodos de energia; arcos; pontes; projeto estrutural; Elementos finitos 


\section{INTRODUCCIÓN}

Esta investigación ha tomado un enfoque de construcción civil y se enfoca en el análisis del arco; puesto que, se entiende su importancia independiente de su aplicación práctica, se reconoce el arco como el mayor invento tensional del arte clásico (Torroja, 1996). Esta forma de construcción ha existido desde los primeros asentamientos humanos; simplemente apoyando una pieza de piedra junto a otra se construye un arco triangular; o formando una estructura escalonada se tiene un arco maya, en la que el arco se cierra por la aproximación progresiva de dos partes de un muro (Sánchez, 2011). Los avances ingenieriles en la construcción de puentes van acompañados en nuevas técnicas de construcción de arcos que aprovechan las ventajas de esta tipología estructural (Rueda, 2014).

Cualquier desviación de la trayectoria del arco implica la aparición de deflexiones para seguir transmitiendo las cargas a los extremos y los apoyos. La magnitud de las deflexiones es proporcional a la desviación (excentricidad) entre el eje del arco y el funicular de cargas (Pérez, 2009). Además, el arco sufre peligros de pandeo. Como elemento estructural a compresión soportan compresiones elevadas. Al ser fijos sus arranques, el arco tiende a tomar una forma de pandeo más resistente, puede fallar por pandeo transversal si no cuenta con apoyo transversal, o por pandeo en el plano cuando su sección es muy esbelta (Torroja, 1996).

De las muchas formas en que un ingeniero puede abordar el problema del cálculo de la deflexión en los arcos, dos fueron usadas en esta investigación para comprobar los resultados: los métodos energéticos, que hacen intervenir una entidad física como el trabajo y la energía de deformación, o la energía potencial total (Young, 1981); el software SAP 2000, programa que cálculo de las deflexiones en arcos por el método de los elementos finitos, herramienta potente que en la actualidad viene remplazando a los métodos tradicionales de cálculo de todo tipo de estructuras.

Esta investigación se propuso determinar la mejor opción, entre métodos energéticos y el método de elementos finitos, para abordar el problema del cálculo de la deflexión en arcos circulares de hormigón para un puente. Para esto se calcularon los desplazamientos lineales y angulares en arcos por los dos métodos y se comparó la deflexión total obtenida por ambos caminos.

La importancia de comparar estos dos métodos recae en la necesidad de optimizar los beneficios que el análisis estructural puede proveer en la construcción de puentes. Cuando un sistema estructural está sometido a carga, se desarrollan acciones y esfuerzos internos, estos últimos producen deformaciones unitarias internas que pueden ser producidas por fuerzas axiales, fuerzas cortantes, momentos de flexión y momentos de torsión que actúan separadamente o en una combinación cualquiera. Para determinar las deformaciones y los desplazamientos de estructuras se debe hacer uso de las relaciones básicas entre esfuerzo, deformaciones unitarias y desplazamientos. Sin embargo, con frecuencia se pueden obtener resultados parecidos en forma más sencilla utilizando los principios de energía (Leontovich, 1987). 
MÉTODO

El estudio se desarrolló bajo un enfoque cuantitativo de corte transversal. Tuvo alcance descriptivo y aplicado, dado que se propuso calcular los desplazamientos lineales y angulares en arcos circulares de hormigón, empleando métodos energéticos y comparando los resultados con los obtenidos al aplicar el software SAP 2000 (que se basa en el método de elementos finitos). El análisis de ambos métodos se desarrolló tomando el caso de un puente llamado Huanpatita ubicado en el departamento de Potosí (Bolivia).

\section{Cálculo del peso propio del arco}

Descomposición del valor g

$$
g^{\prime}=\frac{g}{\cos \theta}-g
$$

Ecuación de la carga

$$
y=k * x^{n}
$$

Área

Centro de gravedad $x$

$$
\frac{n+1}{n+2} a
$$

\section{Diseño de hormigón armado}

Resistencia requerida según $\mathrm{ACI}$

$$
\mathrm{U}=1,40 \mathrm{D}+1,7 \mathrm{~L}
$$




\section{Diseño a flexo compresión}

$\mathrm{Si}$ la columna es susceptible a sufrir grandes deformaciones laterales puede producirse lo que se conoce como falla estabilidad o falla por pandeo, clasificando este tipo de columnas como columnas esbeltas.

$$
\left(\emptyset P_{n}, \emptyset M_{n}\right) \geq\left(P_{u}, M_{u}\right)
$$

Dimensionamiento inicial

$$
A_{s}=\rho A_{g}
$$

La cuantiar óptima de acero para el diseño recomendada por el código ACI es $\rho=0,03$

Siendo e=excentricidad, $M=$ Momento, $N=$ Normal, $\gamma=$ Propiedad de la secion, $h=$ Altura y $d=$ Peralte, se tendrá que escoger la altura conveniente para el prediseño.

$$
e=\frac{M}{N} \quad \text { (9) } \quad \gamma=\frac{d}{h}
$$

El cálculo del ancho será igual a

$$
b=\frac{P_{u}}{\left(\frac{P}{A}\right) * h}
$$

Siendo $(P / A)$ el encontrado en el diagrama de interacción. Mu=Momento ultimo. En primera instancia se realizó el diseño considerando como columna corta.

$$
\frac{M_{u}}{A_{g} h}
$$

$$
\frac{P_{u}}{A_{g}}
$$

As $=$ Acero requerido .

Del diagrama de interacción del código ACI se obtiene la cuantía del acero:

$$
A_{s}=\rho * b * h
$$

Verificación de esbeltez

$$
l_{e}=\beta * l
$$


le=Longitud efectiva, $l=$ longuitud total y $\beta=$ factor de longuitud efectiva

Según Gere (2006), el valor del factor de longitud efectiva es $\beta=0,7$.

Para arcos empotrados

$$
\lambda=\frac{l_{e}}{\sqrt{\frac{I}{A}}}
$$

Siendo $\lambda \leq 35$ columna corta; $35 \leq \lambda \leq 100$ columna esbelta; $\lambda=$ Coeficiente de esbeltes; $\mathrm{y}$ I=Inercia de la seccion transversal.

Refuerzo máximo en miembros a compresión

$$
\frac{A_{s}}{A_{g}} \leq 0,8
$$

Refuerzo mínimo en miembros a compresión

$$
\frac{A_{s} * f_{y}}{A_{g} * f_{c}} \geq 0,35
$$

Incremento del momento debido a esbeltez

$$
\begin{aligned}
& M_{c}=\delta_{b} M_{2 b}+\delta_{s} M_{2 s} \\
& \delta_{b}=\frac{C_{m}}{1-\frac{P_{u}}{\emptyset P_{c r}}} \quad \text { (20) } \quad \delta_{s}=\frac{1}{1-\frac{\sum P_{u}}{\emptyset \sum P_{c r}}}
\end{aligned}
$$

$P u=$ carga axialmayorada

Pcr $=$ carga de pandeo de Euler

$\emptyset=\quad$ factor de resistencia para compresión axial

$M 2 b=$ momento en el elemento comprimido debido a las cargas gravitatorias mayoradas que no provoca desplazamiento lateral apreciable calculado mediante un análisis de pórtico elástico convencional de primer orden, siempre positivo

$\delta 2 b=$ tensión correspondiente a $M 2 b$

$M 2 S=$ momento en un elemento comprimido debido a cargas laterales o gravitatorias mayoradas que provocan un desplazamiento lateral

$\delta 2 S=$ tensión correspondiente a M2S 
$E=\quad$ Modulo de elasticidad

$I=\quad$ Momento de inercia respecto al eje considerado $E I=E c$

$$
P_{c r}=\frac{\pi^{2} E I}{(\beta * \emptyset * R)^{2}}
$$

$$
\begin{gathered}
E I=\frac{\frac{E_{c} I_{g}}{2,5}}{1+\varphi} \\
I_{g}=\frac{b * h^{3}}{12}
\end{gathered}
$$$$
E_{C}=15000 \sqrt{f^{\prime}}
$$

$$
\varphi=\frac{M_{d u}}{M_{u}}
$$

$M d u=$ Menor momento mayorado

$M u=$ Mayor momento mayorado

Con el momento aumentando encontrado se obtiene la cuantía del acero de ACI.

\section{Diseño a corte}

(26)

$$
\begin{gathered}
\mathrm{V}_{\mathrm{u}} \leq \varnothing \mathrm{V}_{\mathrm{n}} \\
\mathrm{V}_{\mathrm{n}}=V_{c}+V_{s}
\end{gathered}
$$

$$
V_{c}=\left(1+\frac{N_{u}}{140 A_{g}}\right) 2 \sqrt{f_{c}^{\prime}} b_{w} d
$$

$$
\mathrm{V}_{\mathrm{u}}=\varnothing V_{c}+\frac{\emptyset A_{v} f_{y} d}{s}
$$

$\emptyset=0,85$ para corte

$$
V_{u}=2 \sqrt{f^{\prime}}{ }_{c} b_{w} d
$$


$\mathrm{Si}$

$$
\mathrm{V}_{\mathrm{u}} \leq \varnothing \mathrm{V}_{c}
$$

Acero mínimo

$$
A_{v}=50 \frac{b_{w} S}{f_{y}}
$$

Deflexiones instantáneas

$$
\Delta_{i u}=\frac{f}{E_{c} I_{u t}}
$$

$$
M_{c r}=\frac{f_{r} I_{g}}{y_{t}}
$$

$$
f_{r}=7,5 \sqrt{f^{\prime}}
$$

Dónde

$$
I_{e}=\left(\frac{M_{c r}}{M_{a}}\right)^{3} I_{g}+\left[1-\left(\frac{M_{c r}}{M_{a}}\right)^{3}\right] I_{c r} \quad y \leq I_{g}
$$

Donde Icr es el momento de inercia para la sección transformada fisurada.

$$
I_{c r}=I_{1}+I_{2}+I_{3}
$$

De la sumatoria de fuerzas se puede encontrar la siguiente ecuación para " $\mathrm{x}$ "

$$
b *\left(\frac{x^{2}}{2}\right)+(n-1) A_{s}(x-c)=n A_{s}^{\prime}(d-x)
$$

Dónde:

$$
n=\frac{E_{s}}{E_{c}}
$$


Se utilizó las siguientes ecuaciones para el cálculo de las inercias

$$
\begin{aligned}
& I_{1}=\frac{b * x^{3}}{12}+b x\left(\frac{x}{2}\right)^{2} \\
& I_{2}=A_{s}(n-1) *(x-c)^{2} \\
& I_{3}=n A_{s} *(d-x)^{2}
\end{aligned}
$$

El cálculo final para la deflexión diferida aplicó la siguiente fórmula

$$
\Delta_{i c}=\frac{f}{E_{c} * I_{e}}
$$

\section{RESULTADOS}

En Bolivia, específicamente en el municipio de Betanzos del departamento de Potosí, se realizó la construcción del puente Huanpatita. Teniendo en el lugar de emplazamiento las características topografías del Gráfico 1 se ve por conveniente la utilización de una estructura en arco.

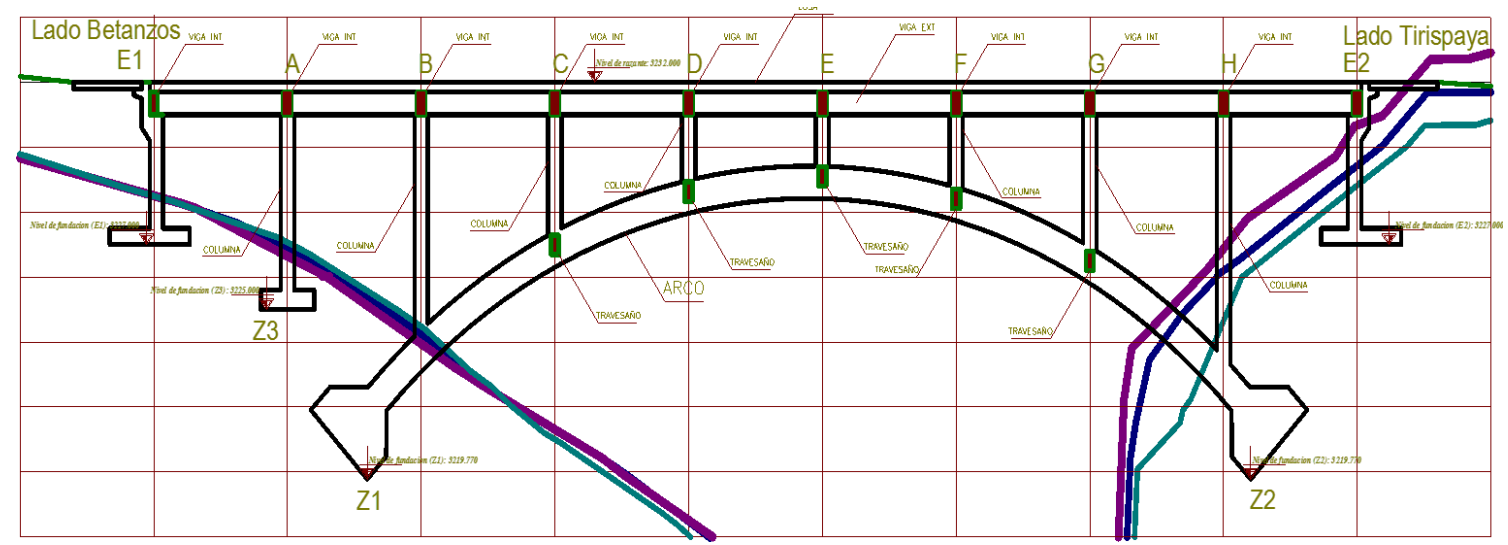

Gráfico 1. Representación de las características topográficas del puente

El gráfico 1 representa un puente que una a Betanzos con Tiquipaya. A continuación, se presenta el procedimiento de pre-dimensionamiento. Según el criterio de diseño que recomienda Torroja (1996) para un arco eficaz la relación de flecha y luz será $f / l=1 / 5$ 
En este caso se considera: $l=32,0 \mathrm{~m} ; f=6,40 \mathrm{~m}$. Con estos datos se calculó los diferentes parámetros geométricos del arco.

$$
\begin{gathered}
R=\frac{l^{2}+4 f^{2}}{8 f} \\
R=\frac{(32 m)^{2}+4(6,40 \mathrm{~m})^{2}}{8(6,40 \mathrm{~m})}=23,2 \mathrm{~m} \\
\operatorname{seno}\left(\frac{\emptyset}{2}\right)=\frac{l}{2 R} \\
\operatorname{seno}\left(\frac{\emptyset}{2}\right)=\frac{32 \mathrm{~m}}{2 * 23,2 \mathrm{~m}} \\
\varnothing=1,522 \mathrm{rad}
\end{gathered}
$$

Peso propio del arco, se procede con el análisis del peso propio del arco de la siguiente forma:

$$
\begin{gathered}
g=2,50 \mathrm{Tn} / \mathrm{m}^{3} *(1 \mathrm{~m} * 0,4 \mathrm{~m})=1,00 \mathrm{Tn} / \mathrm{m} \text {. Usando la ecuación (1) } \\
\qquad \begin{array}{c}
g^{\prime}=\frac{1,00 \mathrm{Tn} / \mathrm{m}^{3}}{\cos \left(\frac{1,522}{2}\right)}-1,00 \mathrm{Tn} / \mathrm{m}^{3} \\
g^{\prime}=0,38 \mathrm{Tn} / \mathrm{m}^{3}
\end{array}
\end{gathered}
$$

Para el análisis para cargas g y g' se usó el áreas y centro de gravedad, ecuaciones (3)

$$
\begin{gathered}
A=P=\frac{16 * 0,38}{0,38+1}=1,65 \mathrm{Tn} \\
\bar{x}=\frac{0,363+1}{0,363+2} 16=9,23 \mathrm{~m}
\end{gathered}
$$

Los resultados obtenidos del cálculo dan: $x[m]=16, \mathrm{z}[\mathrm{m}]=6,4, \mathrm{~N}[\mathrm{Tn}]=22,74, \mathrm{~V}[\mathrm{Tn}]=-$ 1,0100, M[Tn*m]=3,048, Deflexión $[\mathrm{mm}]=-0,31$ 
Peso de las columnas, el peso en las columnas se grafica en la Figura 2

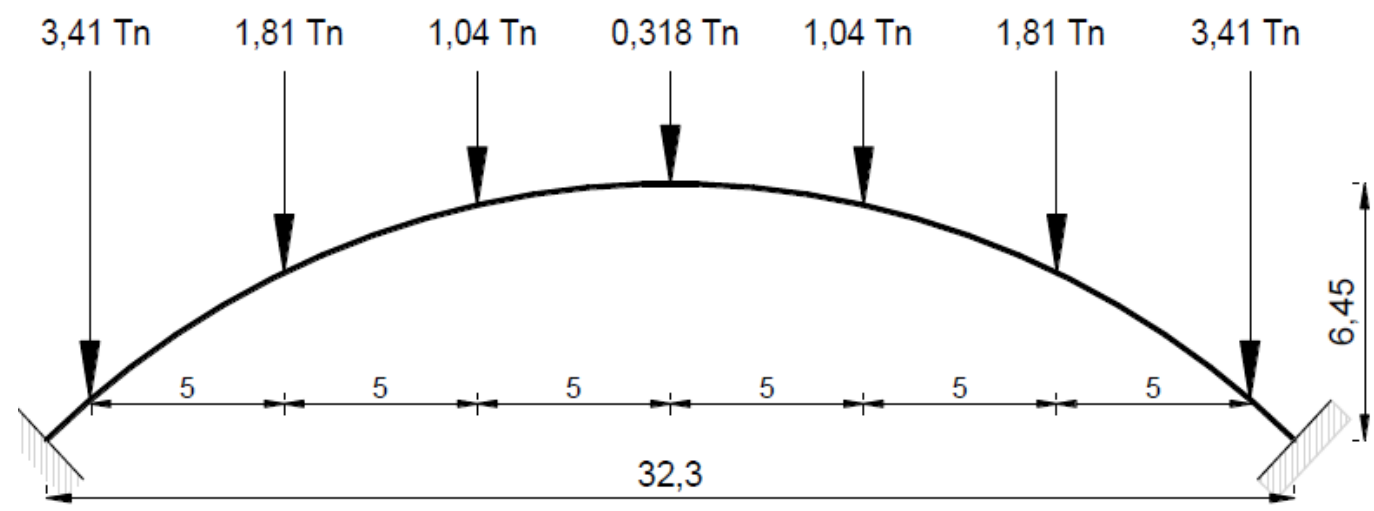

Gráfico 2. Datos para el cálculo del peso en las columnas

Según se observa en la Figura 2, los respectivos pesos de las columnas son los siguientes:

Peso columna $\mathrm{F}=(0,40 * 0,5) * 1,59 * 2,5=0,318 \mathrm{Tn}$

Peso columna E y G $=(0,40 * 0,5) * 2,13 * 2,5=1,035 \mathrm{Tn}$

Peso columna C y G $=(0,40 * 0,5) * 3,38 * 2,5=1,81 \mathrm{Tn}$

Peso columna B y H $=(0,40 * 0,5) * 7,03 * 2,5=3,41 \mathrm{Tn}$

Los resultados obtenidos del cálculo dan: $x[m]=16, \mathrm{z}[\mathrm{m}]=6,4, \mathrm{~N}[\mathrm{Tn}]=-4,19, \mathrm{~V}[\mathrm{Tn}]=-$ 0,1590, M $\left[\mathrm{Tn}^{*} \mathrm{~m}\right]=-1,058$, Deflexión $[\mathrm{mm}]=-0,04$

Análisis de carga muerta estructural $\mathrm{DC}$, el peso baranda es el siguiente: $\mathrm{PB}=$ $(0,159 * 0,2 * 46 * 2,5)+(0,038 * 45,50 * 2,5 * 4)=20,947 \mathrm{Tn}$

Peso bordillo $=2 *[-(0,5 * 0,30 * 0,03)+(0,60 * 0,25)] * 45,30 * 2,5=32,96 \mathrm{Tn}$

Peso acera $=2 *(0,38 * 0,15) * 45,5 * 2,5=43,23 \mathrm{Tn}$

Peso tablero $=(7,3 * 0,3) * 45,5 * 2,5=249,11 \mathrm{Tn}$

Peso viga exterior $=2 *(0,7 * 0,3) * 45,5 * 2,5=47,78 \mathrm{Tn}$

Peso viga interior $=10 *[(5,4 * 0,7)+(1,2 * 0,3)+(0,5 * 0,4 * 1,2)] * 0,30 * 2,5=32,85 \mathrm{Tn}$

$$
\begin{gathered}
\sum \mathrm{P}_{\mathrm{DC}}=(20,94+32,96+43,23+249,11+47,78+32,85) \mathrm{Tn}=426,87 \mathrm{Tn} \\
\mathrm{q}_{\mathrm{DC}}=\frac{\mathrm{P}_{\mathrm{DC}}}{\mathrm{L}_{\mathrm{C}} * \mathrm{~W}} \\
\mathrm{q}_{\mathrm{DC}}=\frac{426,87 \mathrm{Tn}}{2 * 45,5}=4,69 \frac{\mathrm{Tn}}{\mathrm{m}} \\
\mathrm{P}_{\mathrm{DC}}=4,69 \frac{\mathrm{Tn}}{\mathrm{m}} * 5 \mathrm{~m}=23,45 \mathrm{Tn}
\end{gathered}
$$


Peso total en las columnas, se considera el siguiente cálculo:

Columna F Peso columna $\mathrm{F}=0,318 \mathrm{Tn}+23,45 \mathrm{Tn}=23,77 \mathrm{Tn}$

Columna E y G Peso columna E y G=1,035 $\mathrm{Tn}+23,45 \mathrm{Tn}=24,48 \mathrm{Tn}$

Columna D y H Peso columna D y H=1,81 $\mathrm{Tn}+23,45 \mathrm{Tn}=25,26 \mathrm{Tn}$

Columna C y I Peso columna C y I=3,41 Tn+23,45 Tn=26,86 Tn

Obteniendo las cargas para el arco presentadas en el Gráfico 3.

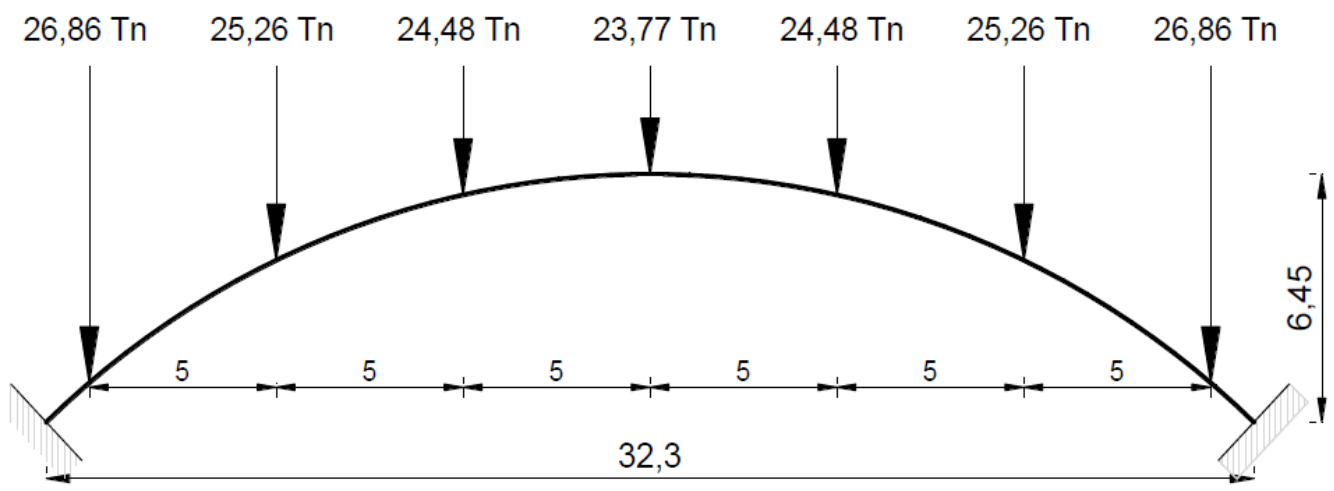

Gráfico 3. Peso total de las columnas

Los resultados obtenidos del cálculo dan: $x[m]=16, \mathrm{z}[\mathrm{m}]=6,4, \mathrm{~N}[\mathrm{Tn}]=-96,77, \mathrm{~V}[\mathrm{Tn}]=-$ 11,840, M[Tn*m]=23,745, Deflexión $[\mathrm{mm}]=-1,64$

Peso total en las columnas, se considera el siguiente cálculo:

El camión de diseño (tren de carga de tipo HL-93), que el típico semitrailer: el eje frontal es de $3.6 \mathrm{~T}$ seguido de $4.3 \mathrm{~m}$ de un eje de $14.8 \mathrm{~T}$ y finalmente el eje posterior de $14.8 \mathrm{~T}$ que está ubicado a una distancia variable de $4.3 \mathrm{~m}$ a $9.0 \mathrm{~m}$.Carga de carril de diseño consiste en una carga distribuida de $9.3 \mathrm{~N} / \mathrm{mm}$ y se asume ocupa una región de $3.0 \mathrm{~m}$ transversalmente.

El caso más desfavorable de carga para el arco será: Peso columna $\mathrm{F}=41,93 \mathrm{Tn}$, Peso columna $\mathrm{E}=19,06 \mathrm{Tn}$, Peso columna $\mathrm{E}=14,40 \mathrm{Tn}$, Peso columna $\mathrm{D}=15,41 \mathrm{Tn}$, Peso columna $\mathrm{H}=14,40 \mathrm{Tn}$, Peso columna $\mathrm{C}=10,08 \mathrm{Tn}$ y Peso columna $\mathrm{I}=10,08 \mathrm{Tn}$.

Los resultados obtenidos del cálculo dan: $x[\mathrm{~m}]=16, z[\mathrm{~m}]=6,4, N[\mathrm{Tn}]=-92,70, V[\mathrm{Tn}]=-$ 19,5570, M[Tn*m]=63,818, Deflexión $[\mathrm{mm}]=-8,22$ 


\section{Diseño del arco}

Se presenta el cálculo para flexo compresión y diseño a corte.

Para el diseño a flexo compresión se considera el siguiente cálculo:

$$
\begin{gathered}
U=1,40 D+1,7 L \\
+M_{u}=1,4(28,49)+1,7(63,818) \mathrm{Tn}-m \\
+M_{u}=148,38 \mathrm{Tn}-\mathrm{m}
\end{gathered}
$$

Para el momento negativo

$$
\begin{gathered}
U=1,40 D+1,7 L \\
-M_{u}=1,4(22,64)+1,7(27,69) \mathrm{Tn}-m \\
-M_{u}=78,77 \mathrm{Tn}-m
\end{gathered}
$$

Para el momento normal

$$
\begin{gathered}
U=1,40 D+1,7 L \\
N_{U}=1,40(165,35)+1,7(111,31) \mathrm{Tn} \\
N_{U}=420,72 \mathrm{Tn}
\end{gathered}
$$

Dimensionamiento de la sección transversal

$$
A_{s}=\rho A_{g}
$$

La cuantiar óptima para el diseño recomendada por el código ACI es $\rho=0,03$

La excentricidad se encontrará con la ecuación (8)

$$
e=\frac{148,38 \mathrm{Tn} * m}{420,72 \mathrm{Tn}}
$$

Da como resultado e $=0,35 \mathrm{~m}$. Considerando una altura inicial de:

$$
\frac{e}{h}=\frac{0,35 m}{1 m}=0,35
$$


Si rec $=5 \mathrm{~cm}$ y $\emptyset=2,5 \mathrm{~cm}$

$$
\begin{gathered}
d=h-\frac{1}{2} \emptyset-r e c \\
d=100 \mathrm{~cm}-\frac{1}{2} 2,5 \mathrm{~cm}-5 \mathrm{~cm} \\
d=93,75 \mathrm{~cm} \\
\gamma=\frac{d}{h} \\
\gamma=\frac{93,75 \mathrm{~cm}}{100 \mathrm{~cm}}=0,93
\end{gathered}
$$

El cálculo del ancho con la ecuación 10 será igual a:

$$
\begin{gathered}
b=\frac{P_{u}}{\left(\frac{P}{A}\right) * h} \\
\frac{P}{A}=1184,3 \mathrm{Tn} / \mathrm{m}^{2} \\
b=\frac{420,72 \mathrm{Tn}}{1184,3 \mathrm{Tn} / \mathrm{m}^{2} * 1 \mathrm{~m}}=0,36 \mathrm{~m} \\
b \cong 0,4 \mathrm{~m}
\end{gathered}
$$

En primera instancia se realizará el diseño con las ecuaciones (11) y (12) considerando como columna corta.

$$
\begin{gathered}
\frac{P_{u}}{A_{a}}=\frac{420,72 \mathrm{Tn}}{0,4 \mathrm{~m} * 1 \mathrm{~m}}=1051,8 \mathrm{Tn} / \mathrm{m}^{2} \\
\frac{P_{u}}{A_{g}}=1,49 \mathrm{klb} / \mathrm{in}^{2} \\
\frac{M_{u}}{A_{g} h}=0,56 \mathrm{~kb} / \mathrm{in}^{2}
\end{gathered}
$$


La cuantía del acero es:

$$
\rho=0,0218
$$

Con la ecuación (13) se obtiene el acero requerido

$$
\begin{gathered}
A_{s}=\rho * b * h \\
A_{s}=0,022 * 100 \mathrm{~cm} * 40 \mathrm{~cm}=88 \mathrm{~cm}^{2}
\end{gathered}
$$

Cálculo de la longitud efectiva ecuación (14)

$$
\begin{gathered}
l_{e}=\beta * \emptyset * R \\
l_{e}=0,7 * 1,522 * 23,2 \mathrm{~m}=24,71 \mathrm{~m}
\end{gathered}
$$

Verificación de la esbeltez, ecuación (15)

$$
\begin{gathered}
\lambda=\frac{l_{e}}{\sqrt{\frac{I}{A}}} \\
\lambda=\frac{24,71 m}{\sqrt{\frac{0,4 m * 1 m^{3} / 12}{0,4 m * 1 m}}}=89,6
\end{gathered}
$$

Se trata de una columna esbelta. Refuerzo máximo en miembros a compresión con la ecuación (16)

$$
\begin{gathered}
\frac{A_{s}}{A_{g}} \leq 0,8 \\
\frac{80 \mathrm{~cm}^{2}}{(100 * 40) \mathrm{cm}^{2}}=0,02 \leq 0,8
\end{gathered}
$$


Refuerzo mínimo en miembros a compresión con la ecuación (17)

$$
\begin{gathered}
\frac{A_{s} * f_{y}}{A_{g} * f_{c}} \geq 1,35 \\
\frac{80 \mathrm{~cm}^{2} * 4200 \mathrm{~kg} / \mathrm{cm}^{2}}{(100 * 40) \mathrm{cm}^{2} * 280 \mathrm{~kg} / \mathrm{cm}^{2}}=0,3 \geq 0,135
\end{gathered}
$$

Incremento del momento debido a esbeltez.

Cálculo de la elasticidad ecuación (23).

$$
E_{C}=15000 \sqrt{280}=250998,01 \mathrm{~kg} / \mathrm{cm}^{2}
$$

Cálculo de la inercia gruesa ecuación (24)

$$
I_{g}=\frac{40 * 100^{3}}{12}=3333333,33 \mathrm{~cm}^{4}
$$

Cálculo de $\varphi$ con la ecuación (25)

$$
\begin{gathered}
M_{d}=148,38 \mathrm{Tn}-m \\
M_{d u}=39,886 \mathrm{Tn}-m \\
\varphi=\frac{39,886 \mathrm{Tn}-\mathrm{m}}{148,38 \mathrm{Tn}-\mathrm{m}} \\
\varphi=0,269
\end{gathered}
$$


Con la ecuación (22)

$$
E I=\frac{\frac{E_{c} I_{g}}{2,5}}{1+\varphi}
$$

$E I=\frac{\frac{250998,01 \frac{\mathrm{kg}}{\mathrm{cm}^{2}} * 3333333,33 \mathrm{~cm}^{4}}{2,5}}{1+0,269}=26372,26 \mathrm{Tn} * \mathrm{~m}^{2}$

Cálculo de la carga crítica ecuación (21).

$$
\begin{gathered}
P_{c r}=\frac{\pi^{2} E I}{(\beta * \emptyset * R)^{2}} \\
P_{c r}=\frac{\pi^{2} 21853,47 \mathrm{Tn} * m^{2}}{(0,7 * 1,522 * 23,2)^{2}}=10530,14 \mathrm{Tn}
\end{gathered}
$$

Utilizando la ecuación (20)

$$
\begin{gathered}
\delta_{b}=\frac{1}{1-\frac{420,717 \text { Tn }}{0,75 * 10530,14 T n}}=1,056 \gg 1 \\
M_{c}=\delta_{b} M_{2 b}
\end{gathered}
$$

Incremento del momento utilizando la ecuación (18)

$$
\begin{gathered}
M_{c}=1,056 *(148,38 \operatorname{Tn} * m) \\
M_{c}=156,73 \mathrm{Tn} * m
\end{gathered}
$$


Cálculo de acero considerando esbeltez con ecuaciones (11) y (12)

$$
\begin{gathered}
\frac{P_{u}}{A_{g}}=\frac{420,720 \mathrm{Tn}}{1 \mathrm{~m} * 0,40 \mathrm{~m}}=1051,8 \mathrm{Tn} / \mathrm{m}^{2} \\
\frac{M_{u}}{A_{g} h}=\frac{156, \mathrm{Tn} * \mathrm{~m}}{0,40 \mathrm{~m} * 1 \mathrm{~m}^{2}}=391,82 \mathrm{Tn} / \mathrm{m}^{2} \\
\frac{P_{u}}{A_{g}}=1,49 \mathrm{klb} / \mathrm{in}^{2} \\
\frac{M_{u}}{A_{g} h}=0,56 \mathrm{klb} / \mathrm{in}^{2}
\end{gathered}
$$

El valor del acero es 88cm². Disposición $18 \emptyset 25$.

Para el diseño a corte compresión se considera el siguiente cálculo:

$$
\begin{gathered}
U=1,40 D+1,7 \mathrm{~L} \\
V_{U}=1,40(5 \mathrm{Tn})+1,7(18,27 \mathrm{Tn}) \\
V_{U}=38,059 \mathrm{Tn} \\
\mathrm{V}_{\mathrm{u}} \leq \emptyset \mathrm{V}_{\mathrm{n}} \\
\mathrm{V}_{\mathrm{n}}=V_{c}+V_{s} \\
\mathrm{~V}_{\mathrm{u}}=\emptyset V_{c}+\frac{\emptyset A_{v} f_{y} d}{s}
\end{gathered}
$$

$\emptyset=0,85$.

Constante que resiste el hormigón con la ecuación (28)

$$
\begin{gathered}
V_{c}=\left(1+\frac{N_{u}}{140 A_{g}}\right) 0,083 \sqrt{f^{\prime}{ }_{c}} b_{w} d \\
V_{c}=\left(1+\frac{420720 \mathrm{~kg}}{140(40 \mathrm{~cm} * 100 \mathrm{~cm})}\right) 0,083 \sqrt{{f^{\prime}}_{c}} b_{w} d \\
V_{c}=9,72 \mathrm{Tn}
\end{gathered}
$$


Como $\mathrm{V}_{\mathrm{u}}>\varnothing \mathrm{V}_{\text {c.. }}$ Se requiere acero de diseño

$$
\begin{gathered}
V_{s}=\frac{V_{u}}{\emptyset}-V_{c} \\
V_{s}=\frac{30,059 \mathrm{Tn}}{0,85}-9,72 \mathrm{Tn} \\
V_{s}=25,64 \mathrm{Tn} \\
s=\frac{A_{v} * f_{y} * d}{V_{s}} \\
a=\frac{A_{s} * f y}{0,85 * f c * b}
\end{gathered}
$$

Siendo $\mathrm{A}_{\mathrm{s}}=80 \mathrm{~cm}^{2}$

$$
\begin{aligned}
& a=\frac{80 * 4200 \mathrm{~kg} / \mathrm{cm}^{2}}{0,85 * 280 \mathrm{~kg} / \mathrm{cm}^{2} * 40 \mathrm{~cm}} \\
& a=35,29 \mathrm{~cm} \\
& d_{v 1}=d-\frac{a}{2} \\
& d_{v 2}=0,72-\frac{h}{10} \\
& d_{v 3}=0,9 * d \\
& d_{v 1}=93,75 \mathrm{~cm}-\frac{35,29 \mathrm{~cm}}{2}=76,11 \mathrm{~cm} \\
& d_{v 2}=0,72 * \frac{1000 \mathrm{~mm}}{10}=72 \mathrm{~cm} \\
& d_{v 3}=0,9 * 93,75 \mathrm{~cm}=84,38 \mathrm{~cm} \\
& d_{v \max }=76,11 \mathrm{~cm} \\
& s=\frac{A_{v} * f_{y} * d}{V_{s}} \\
& A_{V}=2 * 1,13 \mathrm{~cm}^{2}=2,26 \mathrm{~cm}^{2} \\
& s=\frac{2,26 \mathrm{~cm}^{2} * 4200 \frac{\mathrm{kg}}{\mathrm{cm}^{2}} * 93,75 \mathrm{~cm}}{25640 \mathrm{~kg}}=34,7 \mathrm{~cm}
\end{aligned}
$$

Disposición $\emptyset 12$ c/30cm 


\section{Cálculo del momento de fisuración}

$$
f_{r}=2 \sqrt{f^{\prime}}
$$

Se calcula el módulo de rotura con la ecuación (35)

$$
\begin{gathered}
f_{r}=2 \sqrt{280} \\
f_{r}=33,47^{\mathrm{kg} / \mathrm{cm}^{2}}
\end{gathered}
$$

Excentricidad en la clave con la ecuación (8)

$$
\begin{gathered}
e=\frac{M_{u}}{N_{u}} \\
M_{a}=(28,49+63,82) T n-m=92.31 \mathrm{Tn}-m \\
M_{a}=92.31 \mathrm{Tn}-m \\
N_{a}=(134,57+66,64) \mathrm{Tn}=201,21 \mathrm{Tn} \\
N_{a}=201,21 \mathrm{Tn} \\
e=\frac{92.31 \mathrm{Tn}-\mathrm{m}}{201,21 \mathrm{Tn}} \\
e=0,46 \mathrm{~m} \\
y_{t}=50 \mathrm{~cm} \\
I_{g}=\frac{b * h^{3}}{12} \\
I_{g}=\frac{40 \mathrm{~cm} *(100)^{3}}{12}=3333333,33 \mathrm{~cm}^{4}
\end{gathered}
$$


Cálculo del momento crítico según la ecuación (34)

$$
\begin{gathered}
M_{c r}=\frac{33,47^{\mathrm{kg}} / \mathrm{cm}^{2} * 4166666,67 \mathrm{~cm}^{4}}{50 \mathrm{~cm}} \\
M_{c r}=22,31 \mathrm{Tn}-\mathrm{m}
\end{gathered}
$$

\section{Cálculo deflexiones instantáneas}

$$
M a=92,308 \mathrm{Tn}-m
$$

Para el cálculo de la inercia efectiva con la ecuación (37)

$$
I_{e}=\left(\frac{M_{c r}}{M_{a}}\right)^{3} I_{g}+\left[1-\left(\frac{M_{c r}}{M_{a}}\right)^{3}\right] I_{c r}
$$

Calculo inercia critica ecuación (38).

$$
I_{c r}=I_{1}+I_{2}+I_{3}
$$

Según el Gráfico 4 se calcula el valor de X usando la ecuación (39)

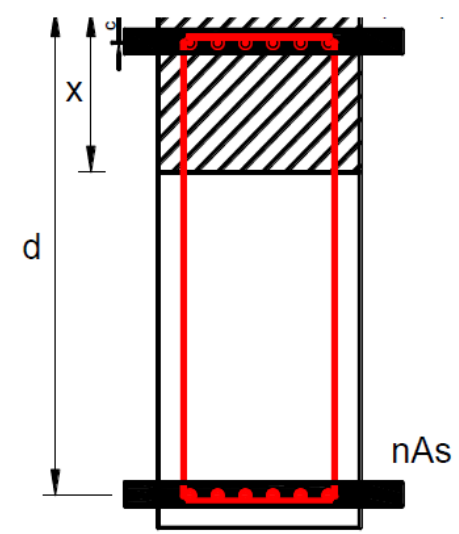

Gráfico 1. Cálculo deflexión instantánea, valor de X 


$$
\begin{gathered}
b *\left(\frac{x^{2}}{2}\right)+(n-1) A_{s}^{\prime}(x-c)=n A_{s}(d-x) \\
n=\frac{E_{s}}{E_{c}} \\
n=\frac{2100000}{15000 \sqrt{280}}=8,37 \\
(n-1) A^{\prime}{ }_{s}=(8,37-1) * 29,45 \mathrm{~cm}^{2} \\
(n-1) A^{\prime}{ }_{s}=328,69 \mathrm{~cm}^{2} \\
n A_{s}=8,37 * 58,91 \mathrm{~cm}^{2} \\
n A_{s}=410,86 \mathrm{~cm}^{2} \\
40 *\left(\frac{x^{2}}{2}\right)+328,69 \mathrm{~cm}^{2}(x-7,05)=410,86 \mathrm{~cm}^{2}(93,75-x) \\
x=30,33 \mathrm{~cm}^{2}
\end{gathered}
$$

Cálculo de las inercias según las ecuaciones (41), (42) y (43)

$$
\begin{gathered}
I_{1}=\frac{b * x^{3}}{3} \\
I_{2}=n A_{s} *(d-x)^{2} \\
I_{3}=(n-1) A_{s}^{\prime} *(x-c)^{2} \\
I_{1}=\frac{40 \mathrm{~cm} *(30,33 \mathrm{~cm})^{3}}{3} \\
I_{1}=372011,16 \mathrm{~cm}^{4} \\
I_{2}=410,86 \mathrm{~cm}{ }^{2} *(93,75 \mathrm{~cm}-30,33 \mathrm{~cm})^{2} \\
I_{2}=1652518,53 \mathrm{~cm}^{4} \\
I_{3}=328,69 *(30,33-7,05)^{2} \\
I_{3}=178136,31 \mathrm{~cm}^{4} \\
I_{e}=\left(\frac{22,31 \mathrm{Tn}-m}{92,308 \mathrm{Tn}-m}\right)^{3} 3333333,3 \mathrm{~cm}+\left[1-\left(\frac{22,31 \mathrm{Tn}-m}{92,308 \mathrm{Tn}-\mathrm{m}}\right)^{3}\right] 2202666 \mathrm{~cm}^{4} \\
I_{e}=0,022 \mathrm{~m}^{4} \\
I_{c r}=2202666 \mathrm{~cm}^{4} \\
I_{c r}=1652518,53 \mathrm{~cm}^{4}+1444757,7 \mathrm{~cm}^{4}
\end{gathered}
$$


Cálculo deflexión instantánea ecuación (44)

$$
\begin{gathered}
\Delta_{i p}=\frac{0,31 \mathrm{~mm} * 0,033 \mathrm{~m}^{4}}{0,022 \mathrm{~m}^{4}}=0,47 \mathrm{~mm} \\
\Delta_{i c}=\frac{0,04 \mathrm{~mm} * 0,033 \mathrm{~m}^{4}}{0,022 \mathrm{~m}^{4}}=0,06 \mathrm{~mm} \\
\Delta_{i p u}=\frac{1,64 \mathrm{~mm} * 0,033 \mathrm{~m}^{4}}{0,035 \mathrm{~m}^{4}}=2,46 \mathrm{~mm} \\
\Delta_{i v}=\frac{8,22 \mathrm{~mm} * 0,033 \mathrm{~m}^{4}}{0,022 \mathrm{~m}^{4}}=12.33 \mathrm{~mm}
\end{gathered}
$$

\section{Cálculo de las flechas referidas}

$$
\lambda=\frac{\xi}{1+50 \rho^{\prime}}
$$

Donde $\rho^{\prime}=A^{\prime} s / b d$ y $\xi$ es el coeficiente dependiente del tiempo y además de la propiedad del material de depender de las características del flujo plástico y de retracción de fraguado.

Con la ecuación (7) se calcula $\rho^{\prime}=A^{\prime} s$

$$
\begin{gathered}
A_{s}^{\prime}=\text { acero en comprecion } \\
A_{s}^{\prime}=N^{\circ}\left(\frac{d^{2}}{4} \pi\right) \\
A_{s}^{\prime}=6\left(\frac{(2,5)^{2}}{4} \pi\right)=29,45 \mathrm{~cm}^{2} \\
\rho=\frac{A_{s}^{\prime}}{b * h} \\
\rho=\frac{29,45 \mathrm{~cm}^{2}}{40 \mathrm{~cm} * 100 \mathrm{~cm}} \\
\rho=0,0074
\end{gathered}
$$


El acero a compresión genera los siguientes valores> a 3 meses 1, a 6 meses 1.2, a 12 meses 1.4, de 5 años a más 2.0.

Considerando 3 meses como tiempo de ejecución de arco, columna y tablero. Ase calcula la deflexión diferida para el peso propio.

$$
\begin{aligned}
& \Delta_{d p}=\left(\frac{1}{1+(50 * 0,0074)}\right) * \Delta_{i p}=0,73 \mathrm{~mm} \\
& \Delta_{(d c)}=\left(\frac{1}{1+(50 * 0,0074)}\right) * \Delta_{i p}=0,22 \mathrm{~mm} \\
& \Delta_{i p u}=\left(\frac{1}{1+(50 * 0,0074)}\right) * \Delta_{i p}=4,48 \mathrm{~mm} \\
& \Delta_{i v}=\left(\frac{1}{1+(50 * 0,0074)}\right) * \Delta_{i p}=12,05 \mathrm{~mm}
\end{aligned}
$$

La deflexión total será:

$$
\begin{gathered}
y=(0,47+0,06+2,46+12,33+0,73+0,22+4,48+12,05) \mathrm{mm} \\
y=32.8 \mathrm{~mm}
\end{gathered}
$$

\section{Control de deflexiones permisibles en arcos circulares}

Las deflexiones máximas permitidas por el código ACI son:

$$
f_{\text {adm }}=\frac{l}{480}
$$

$$
l=\emptyset * R
$$

$$
\begin{aligned}
& f_{\text {adm }}=\frac{35,31}{480}=73,56 \mathrm{~mm} \\
& f_{\text {adm }}>y
\end{aligned}
$$

Se realizó una comparación entre los resultados obtenidos aplicando los métodos energéticos y el Programa SAP 2000. El programa SAP 2000 arroja los siguientes resultados: Peso muerto -0,31; Columnas - 0,04 ; Peso puente -1,64; Carga viva -8,22 
Deflexión total con programa SAP 2000

$$
\Delta=1,28 \mathrm{~mm}+0,09 \mathrm{~mm}+3,92 \mathrm{~mm}+7,19 \mathrm{~mm}=12,48 \mathrm{~mm}
$$

Deflexión total con métodos energéticos

$$
\Delta=0,31 \mathrm{~mm}+0,04 \mathrm{~mm}+1,64 \mathrm{~mm}+8,22 \mathrm{~mm}=10,21 \mathrm{~mm}
$$

Los resultados que se obtuvo por métodos energéticos son mucho menores (2,27 mm) a los obtenidos con el programa Sap2000. Demuestra la eficiencia de los métodos energético en el cálculo de deflexiones en estructuras curvas y en este caso particular que son los arcos circulares.

\section{CONCLUSIONES}

Si bien el método de elementos finitos permite realiza el análisis y cálculo de las deflexiones en arcos, requiere el uso del software estructural SAP 2000, porque la complejidad del cálculo no se acomoda a herramientas tradicionales. Por su parte, los métodos energéticos desarrollados es este trabajo son más sencillos y permiten realizar el análisis y cálculo de deflexiones en arcos usando la herramienta Excel.

Los métodos energéticos son una atractiva opción para la resolución de arcos hiperestáticos y cálculo de deflexiones en estos, ya que se ajustan a secciones curvas; sin embargo, la bibliografía disponible se enfoca más en el análisis estructural de vigas y columnas. Sin embargo, los resultados obtenidos prueban que, haciendo una comparación, los métodos energéticos proveen mejores resultados que el método de elementos finitos; específicamente en el cálculo de deflexiones en arcos circulares. La deformación con métodos energéticos es menor debido a que se toma en cuenta la curvatura del arco; en cambio, el programa estructural SAP 2000 analiza pequeñas secciones de barras rectas que conforman el arco.

En este caso, se realizó el diseño del arco como si este fuera una columna, debido a que el arco de hormigo diseñado trabaja a flexo-compresión, porque el arco que se consideró es circular. En cambio, si se quiere reducir los momentos producidos en el arco por su peso propio y diferentes cargas aplicadas, se debe modificar el arco que para este tome una forma parabólica, disminuyendo significativamente los momentos y reduciendo la cantidad de acero que se usa.

Puesto que la metodología que se siguió para el análisis expuesto en este artículo ha sido descrita a detalle y es replicable, este documento se constituye en una guía base para posteriores investigaciones del área de diseño estructural; para los investigadores que deseen entender la fundamentación por la cual parte la construcción de estructuras en arco.

\section{REFERENCIAS}

ACI. (2005). Requisitos de reglamento para concreto estructural $y$ comentario. In A. 318S-05

Gere, J. (2006). Mecánica de materiales. $6^{\underline{a}}$ edición. Pearson

Leontovich, V. (1987). Pórticos y arcos "Solución para análisis estructural", Cia Editorial Continental

Pérez, U. (2009). Análisis y diseño de arcos. Tesis. México 
Rueda, G. L. (2014). Diseño, construcción, ensayo y análisis de modelos reducidos de estructuras de hormigón armado arco rígido triarticulados, Valencia. I.S.B.N. Ediciones de la UCLM

Sánchez, N. (2011). Geometría de los arcos "guía para la construcción y trazado de los arcos”, España, 1aㅡ edición, región de Murcia
Torroja, M. (1996), Razón y ser de los tipos estructurales, Madrid, $8^{\underline{a}}$ edición, Instituto de Ciencias la Construcción Young, R. (1981). Untying the text: A poststructuralist reader (p. 48). Boston: Routledge \& Kegan Paul 